\title{
Critical Assessment of Microarray Data Analysis
}

National Cancer Institute

\section{Source}

National Cancer Institute. Critical Assessment of Microarray Data Analysis. NCI

Thesaurus. Code C54720.

A group founded to provide a forum to critically assess different techniques used in microarray data mining. It aims to establish the state-of-the-art in microarray data mining, as well as identify progress and highlight the direction for future effort. To achieve these goals, CAMDA adopted the approach of community-wide experiment, letting the scientific community analyze the same standard data sets. Researchers worldwide are invited to take the CAMDA challenge. Results of analyses are discussed and compared at an annual CAMDA conference. 